\author{
Matteo Bassetti \\ Elda Righi \\ Filippo Ansaldi \\ Maria Merelli \\ Cecilia Trucchi \\ Gennaro De Pascale \\ Ana Diaz-Martin \\ Roberto Luzzati \\ Chiara Rosin \\ Leonel Lagunes \\ Enrico Maria Trecarichi \\ Maurizio Sanguinetti \\ Brunella Posteraro \\ Jose Garnacho-Montero \\ Assunta Sartor \\ Jordi Rello \\ Giorgio Della Rocca \\ Massimo Antonelli \\ Mario Tumbarello
}

\section{Erratum to: A multicenter study of septic shock due to candidemia: outcomes and predictors of mortality}

Published online: 3 July 2014

(C) Springer-Verlag Berlin Heidelberg and ESICM 2014

The online version of the original article can be found under doi:10.1007/s00134-014-3310-z.

M. Bassetti · E. Righi · M. Merelli

Infectious Diseases Division, Santa Maria Misericordia University Hospital, Udine, Italy

F. Ansaldi · C. Trucchi

Department of Health Sciences, IRCCS San Martino IST,

University of Genoa, Genoa, Italy

G. De Pascale $\cdot$ M. Antonelli

Critical Care Department, Università Cattolica del Sacro Cuore, Rome, Italy

A. Diaz-Martin · J. Garnacho-Montero

Critical Care and Emergency Clinical Unit, Virgen del Rocio

University Hospital, Seville, Spain

R. Luzzati · C. Rosin

Infectious Diseases Unit, University Hospital of Trieste, Trieste, Italy

L. Lagunes $\cdot$ J. Rello

Critical Care Department, Vall d'Hebron University Hospital, CIBERES, Universitat autonoma de Barcelona, Barcelona, Spain

E. M. Trecarichi - M. Tumbarello

Institute of Infectious Diseases, Università Cattolica del Sacro

Cuore, Rome, Italy

\section{Sanguinetti}

Institute of Microbiology, Università Cattolica del Sacro Cuore, Rome, Italy

\section{B. Posteraro}

Institute of Public Health (Section of Hygiene), Università Cattolica del Sacro Cuore, Rome, Italy

A. Sartor

Microbiology Unit, Santa Maria Misericordia University Hospital, Udine, Italy

G. D. Rocca

Critical Care Department, Santa Maria Misericordia University Hospital, Udine, Italy

\section{Bassetti ( $\bullet$}

Clinica Malattie Infettive, Azienda Ospedaliera Universitaria Santa Maria della Misericordia, Piazzale Santa Maria della Misericordia 15, 33100 Udine, Italy e-mail: mattba@tin.it Tel.: 39-4-32559355

\section{Erratum to: Intensive Care Med (2014) 40:839-845 DOI 10.1007/s00134-014-3310-z}

The given name and family name of the fifth author, Cecilia Trucchi, were transposed; this mistake is corrected here. The authors apologize for any inconvenience. 